\title{
ISOMETRIC IMIMERSIONS OF COMPLETE RIEMANNIAN MANIFOLDS INTO EUCLIDEAN SPACE
}

\author{
CHRISTOS BAIKOUSIS AND THEMIS KOUFOGIORGOS
}

\begin{abstract}
Let $M$ be a complete Riemannian manifold of dimension $n$, with scalar curvature bounded from below. If the isometric immersion of $M$ into euclidean space of dimension $n+q, q<n-1$, is included in a ball of radius $\lambda$, then the sectional curvature $K$ of $M$ satisfies $\lim \sup _{M} K>\lambda^{-2}$. The special case where $M$ is compact is due to Jacobowitz.
\end{abstract}

Generalizing results by Tompkins, Chern and Kuiper, and Otsuki, Jacobowitz proved that a compact $n$-dimensional Riemannian manifold whose sectional curvatures are everywhere less than constant $\lambda^{-2}$ cannot be isometrically immersed into euclidean space of dimension $2 n-1$ so as to be contained in a ball of radius $\lambda$ (see [1] and the references therein). In this note we shall prove a quantitative result concerning isometric immersions, which includes Jacobowitz's theorem as a special case.

The proof of our result will consist in a simple application of a theorem by Omori [3], which we now formulate.

Let $M$ be a complete Riemannian manifold with sectional curvature bounded from below; consider a smooth function $f: M \rightarrow R$ with $\sup f<\infty$. For any $\varepsilon>0$ there exists a point $p \in M$ where $\|\operatorname{grad} f\|<\varepsilon$ and $\nabla^{2} f(X, X)<\varepsilon$ for all unit vectors $X \in T_{p} M$. By $\nabla^{2} f$ we mean the Hessian form of $f$, defined by $\nabla^{2} f(X, Y)=$ $\left\langle\nabla_{X} \operatorname{grad} f, Y\right\rangle$.

THEOREM 1. Let $M$ be a complete n-dimensional Riemannian manifold with scalar curvature $R$ bounded from below. Assume that there exists an isometric immersion $\varphi$ of $M$ into euclidean space of dimension $n+q, q<n-1$, so that $\varphi(M)$ is included in a ball of radius $\lambda$. Then lim $\sup _{M} K \geqslant \lambda^{-2}$, where $K$ is the sectional curvature of $M$.

COROllary. A complete two-dimensional Riemannian manifold, immersed isometrically into euclidean three-space, and whose Gaussian curvature $K$ satisfies $-\infty<-a^{2}$ $<K<0$, is extrinsically unbounded.

Proof of the Theorem. If $n=2$ then $R=2 K$ and we have inf $K>-\infty$. If $n>2$ and inf $K=-\infty$, then inf $R>-\infty$ easily implies sup $K=+\infty$ and the theorem follows. We may therefore assume inf $K>-\infty$.

We shall apply Omori's theorem to the "distance" function $F=\langle\varphi, \varphi\rangle / 2 ; \varphi$ is considered here as tangent vector in euclidean space $E^{n+q}$. By assumption, we have

Received by the editors June 11, 1979.

AMS (MOS) subject classifications (1970). Primary 53C40.

Key words and phrases. Isometric immersion, scalar curvature, sectional curvature, complete Riemannian manifold. 
$\|\varphi\| \leqslant \lambda$ and $f \leqslant \lambda^{2} / 2$, taking the origin to be the center of the ball wherein $\varphi(M)$ lies. Therefore, to any natural number $m$, there exists a point $p_{m} \in M$ where $\nabla^{2} f(X, X)<1 / m$ for all $X \in T p_{m} M$ with $\|X\|=1$. In order to compute the Hessian of $f$, we identify every tangent vector $X$ with $\varphi_{*}(X)$ and obtain $\nabla_{X}^{\prime} \varphi=X$, where $\nabla^{\prime}$ denotes the connection of $E^{n+q}$. Now using this and the Gauss formula, we compute easily $\nabla^{2} f(X, Y)=\langle X, Y\rangle+\langle L(X, Y), \varphi\rangle$, where $L$ stands for the second fundamental form of the immersion. Thus at $p_{m}$ and for every nonzero $X \in T p_{m} M$ we have $1+\langle L(X, X), \varphi\rangle \cdot\|X\|^{-2}<m^{-1}$, hence

$$
\lambda^{-1}\left(1-m^{-1}\right)<\|L(X, X)\| \cdot\|X\|^{-2} \text {. }
$$

From (*) we conclude that, at $p_{m} \in M$, we have $L(X, X) \neq 0$ for $X \neq 0$. Now we use, as in [1], a well-known algebraic lemma [2, p. 28]. Let $L: R^{n} \times R^{n} \rightarrow R^{q}$ be symmetric, bilinear and satisfy $L(X, X) \neq 0$ for $X \neq 0$; if $q<n-1$, there exist linearly independent $X, Y$ so that $L(X, Y)=0$ and $L(X, X)=L(Y, Y)$. We pick two such vectors $X, Y$ in $T p_{m} M$, apply (*) and obtain

$$
\begin{aligned}
\lambda^{-2}\left(1-m^{-1}\right)^{2} & <\|L(X, X)\| \cdot\|L(Y, Y)\| \cdot\|X\|^{-2} \cdot\|Y\|^{-2} \\
\leqslant & \left(\langle L(X, X), L(Y, Y)\rangle-\|L(X, Y)\|^{2}\right) \cdot\left(\|X\|^{2}\|Y\|^{2}-\langle X, Y\rangle^{2}\right)^{-1} .
\end{aligned}
$$

By the Gauss equation, the rightmost term in these inequalities is the sectional curvature of $M$ at $p_{m}$ for the plane spanned by $X$ and $Y$. Now letting $m$ go to infinity, we deduce $\lambda^{-2} \leqslant \lim \sup _{m} K(X \wedge Y)$ and thus prove the theorem.

It is noteworthy that the above proof includes a generalization of the following well-known result. If a compact hypersurface $M$ in $E^{N}$ is contained in a ball of radius $\lambda$, then there exists a point on $M$ where all the normal curvatures are in absolute value not less than $\lambda^{-1}$. For a submanifold $M$ of $E^{N}$, of arbitrary codimension, we define the absolute normal curvature at a point $p \in M$ and in the direction $X \in T_{p} M,\|X\|=1$, to be $\|L(X, X)\|$. Let

$$
C(p)=\min \left\{\|L(X, X)\| / X \in T_{p} M \text { and }\|X\|=1\right\} .
$$

THEOREM 2. Let $M$ be a complete submanifold of $E^{N}$ with sectional curvature bounded from below. If $M$ is contained in a ball of radius $\lambda$, then $\lim \sup _{p \in M} C(p)>$ $\lambda^{-1}$

Proof. Apply Omori's theorem as in Theorem 1 to $\langle\varphi, \varphi\rangle / 2$. From inequality (*) we immediately obtain the conclusion.

We wish to thank D. Koutroufiotis for his aid in this work.

\section{REFERENCES}

1. H. Jacobowitz, Isometric embedding of a compact Riemannian manifold into euclidean space, Proc. Amer. Math. Soc. 40 (1973), 245-246.

2. S. Kobayashi and N. Nomizu, Foundations of differential geometry. Vol. II, Interscience Tracts in Pure and Appl. Math., no. 15, Interscience, New York, 1969.

3. H. Omori, Isometric immersions of Riemannian manifolds, J. Math. Soc. Japan 19 (1967), 205-214.

Department of Mathematics, University of IoAnNina, IonNnina, Grebce 